\title{
C-fiber-related EEG-oscillations induced by laser radiant heat stimulation of capsaicin-treated skin
}

\author{
Claudia Domnick' \\ Michael Hauck ${ }^{1,2,3}$ \\ Kenneth L Casey ${ }^{3}$ \\ Andreas K Engel' \\ Jürgen Lorenz',3,4 \\ 'Department of Neurophysiology \\ and Pathophysiology; ${ }^{2}$ Department of \\ Neurology, University Medical Center \\ Hamburg-Eppendorf, Hamburg, \\ Germany; ${ }^{3}$ Department of Neurology, \\ University of Michigan, Ann Arbor, \\ MI, USA; ${ }^{4}$ Faculty of Life Sciences, \\ Hamburg University of Applied \\ Sciences, Hamburg, Germany
}

\begin{abstract}
Nociceptive input reaches the brain via two different types of nerve fibers, moderately fast A-delta and slowly conducting C-fibers, respectively. To explore their distinct roles in normal and inflammatory pain we used laser stimulation of normal and capsaicin treated skin at proximal and distal arm sites in combination with time frequency transformation of electroencephalography (EEG) data. Comparison of phase-locked (evoked) and non-phase-locked (total) EEG to laser stimuli revealed three significant pain-related oscillatory responses. First, an evoked response in the delta-theta band, mediated by A-fibers, was reduced by topical capsaicin treatment. Second, a decrease of total power in the alpha-to-gamma band reflected both an A- and C-nociceptor-mediated response with only the latter being reduced by capsaicin treatment. Finally, an enhancement of total power in the upper beta band was mediated exclusively by $\mathrm{C}$-nociceptors and appeared strongly augmented by capsaicin treatment. These findings suggest that phase-locking of brain activity to stimulus onset is a critical feature of A-delta nociceptive input, allowing rapid orientation to salient and potentially threatening events. In contrast, the subsequent $\mathrm{C}$-nociceptive input exhibits clearly less phase coupling to the stimulus. It may primarily signal the tissue status allowing more long-term behavioral adaptations during ongoing inflammatory events that accompany tissue damage.
\end{abstract}

Keywords: C-fibers, oscillations, EEG, laser, capsaicin, inflammatory pain

\section{Introduction}

The perception of cutaneous pain arises from the activation of two sets of peripheral sensors. These are thinly myelinated A- and unmyelinated C-nociceptors, which have medium $(5-25 \mathrm{~m} / \mathrm{s})$ and slow $(0.5-2 \mathrm{~m} / \mathrm{s})$ nerve conduction velocities, respectively. ${ }^{1}$ Aside from their different temporal characteristics by inducing first and second pain reactions following a single brief noxious stimulus, ${ }^{2} \mathrm{~A}$ - and C-nociceptors possess different neurochemical transduction properties pointing to likely different roles in normal and pathological pain phenomena. ${ }^{3,4}$ Polymodal C-nociceptors are robustly activated and sensitized by capsaicin, the pungent ingredient of the hot chilli pepper, that acts via the transient receptor potential vanilloid receptor 1 (TRPV1) and is involved in heat hyperalgesia and allodynia during tissue inflammation. ${ }^{5}$ On the other hand, first pain sensation mediated by A-nociceptors, referred to type II AMH with mechanical and heat sensitivity by several authors, is primarily desensitized by moderate to high doses of topical capsaicin whereas (purely mechanical) pinprick pain appears to be mediated by capsaicin-insensitive A-nociceptors. ${ }^{6}$ Our group has demonstrated by positron emission tomography (PET) in healthy volunteers that capsaicin-induced neurogenic inflammation and heat allodynia recruit a different network of supraspinal structures compared to normal heat pain. ${ }^{7}$ However, the differential role of C- and A-nociceptors for inflammatory and normal heat pain has remained unclear due to the low temporal resolution of the PET technique.

Electroencephalography (EEG), as a method of high temporal resolution, has been used in the past in combination with infrared laser stimuli to distinguish between A- and 
C-nociceptor activities. However, although psychophysical studies $^{8}$ demonstrate a double sensation composed of first and second pain following laser stimuli, so-called ultralate components of laser-evoked potentials (LEP) that are related to $\mathrm{C}$-fiber activity can only be elicited when the occurrence of late A-fiber mediated components is avoided, for example by applying a pressure blockade to the nerve. ${ }^{9}$ However, this method is complicated and can only be applied to small skin territories of distal nerve branches. Other methods to selectively activate $\mathrm{C}$-fibers is to stimulate small skin areas, due to the higher regional density of C-nociceptors compared with A-nociceptors, ${ }^{10-13}$ or to stimulate large skin areas at low intensities. ${ }^{14}$

Most EEG and magnetoencephalography (MEG) studies used the standard evoked potential/field method in the timedomain analyzing activity of low frequency bandwidths that are phase-locked to the painful stimuli. However, sensory, motor, and cognitive processes related to discrete stimulus events can also induce power augmentations or reductions of neural oscillations that are time-locked, but not phaselocked to stimulus onset. ${ }^{15}$ If such oscillations are induced by repetitive stimuli without a consistent phase-coupling to the stimulus, they will disappear when averaged in the time domain. To preserve these oscillatory components, the data are first transformed into the frequency domain and then averaged resulting in the total time frequency representation (TFR). A generally low degree of phase-locking is known for C-fiber responses following laser stimuli. For example, Bromm and Treede ${ }^{9}$ applied a latency correction technique to raw traces before averaging to compensate for latency jitter across trials.

Non-phase-locked responses to laser skin stimulation were first demonstrated by Mouraux and colleagues. ${ }^{16}$ These authors showed an increase in signal amplitude in the delta band after stimulus onset and a decrease in the alpha band after $500 \mathrm{~ms}$, the latter comprising correlates of both A- and $\mathrm{C}$-fiber activity as judged by means of latency and reaction time criteria. The induction of activity in higher frequency bands by pain stimuli has been considered in several recent studies, ${ }^{17-19}$ suggesting a relation between gamma band oscillations (above $30 \mathrm{~Hz}$ ) and pain perception. Oscillations in this frequency range are involved in numerous cortical processes including cognitive binding, ${ }^{20}$ muscle-motor coupling,,${ }^{21}$ and tactile spatial attention. ${ }^{22}$

To extend the study of C-fiber-related EEG activity by analysis of higher frequency bands and explore the effect of neurogenic inflammation, we used a multi-taper-method for time frequency transformation of non-phase-locked and phase-locked oscillations after painful stimulation of both normal and capsaicin-treated skin at the volar arm. Nerve conduction velocities of nociceptor activations underlying these oscillations were estimated by referring the distance between proximal and distal stimulation sites to corresponding latency differences.

\section{Methods}

\section{Subjects}

The local ethics review boards of both the Veterans Affairs Hospital and the University of Michigan approved the protocol and the informed consent procedures. Sixteen healthy test subjects ( 8 male, 8 female; mean age 23.2, SD 4.7 years, all right handed) participated in this study after written informed consent and were free to terminate the experiment at any time.

\section{Stimulation, procedure, and pain rating}

We delivered brief infrared laser stimuli of $1 \mathrm{~ms}$ duration and a beam diameter of $5 \mathrm{~mm}$ to proximal and distal sites of the volar sides of left and right arms using a Thulium YAG laser (wavelength $1.96 \mu \mathrm{m}$; Wavelight, Starnberg, Germany). Before the experiment, subjects were made familiar with the use of the rating scale ranging from 0 (no sensation) to 100 (maximal pain), a value of 30 indicating the beginning of a stinging or burning pain sensation. Pain threshold was tested at the dorsum of the left hand by computing the average intensity at which subjects reported first a rating above 30 in three ascending and a rating value below 30 in three descending series of laser stimuli using successive intensity increments of $30 \mathrm{~mJ}$. Before the experiment, one arm (left or right balanced over subjects) was treated proximally as well as distally with topical capsaicin. Capsaicin (8-Methyl-N-Vanillyl-6Nonenamid; Sigma Aldrich, St. Louis, MO) was dissolved in $70 \%$ ethyl alcohol to give a $0.1 \%$ solution. The filter paper $(5 \times 5 \mathrm{~cm})$ saturated with this solution was taped firmly to the respective sites for $30 \mathrm{~min}$. After the exposure, any residuals from the capsaicin solution were removed from the skin by a gauze pad. On the nontreated side, labeled 'normal side,' the same filter paper was attached using the same solution without capsaicin. The stimulation areas were marked with a black crayon for identification of the treated skin areas. Each of the four stimulus sites was tested twice such that the entire session consisted of eight blocks. It started at the proximal untreated skin of the upper arm and proceeded with the untreated skin of the distal forearm. During the third block the stimulation site was changed to the capsaicin-sensitized skin at distal and then at proximal sites. Repetitions of all sites were then done in the reverse order to minimize confounding of site 
with habituation effects over time. One experimental block contained 20 laser pulses of high $(600 \mathrm{~mJ})$ and 20 pulses of low intensity $(450 \mathrm{~mJ})$ in pseudo-random order with variable inter-stimulus intervals (10-2 s). Three seconds after each laser stimulus an acoustic event $(2000 \mathrm{~Hz}$ tone) prompted the verbal rating. After each block the subjects were asked to rate the overall unpleasantness for all pain stimuli using the 100-point scale without an anchor. The total duration of one block was approximately 15-20 minutes. Figure 1 illustrates the test procedure in this paradigm.

\section{Data acquisition and analysis of EEG}

The 64 channel EEG (10\% system QUICK CAP) was digitally recorded using AQUIRE ${ }^{\circledR}$ software (Compumedics Neuroscan,
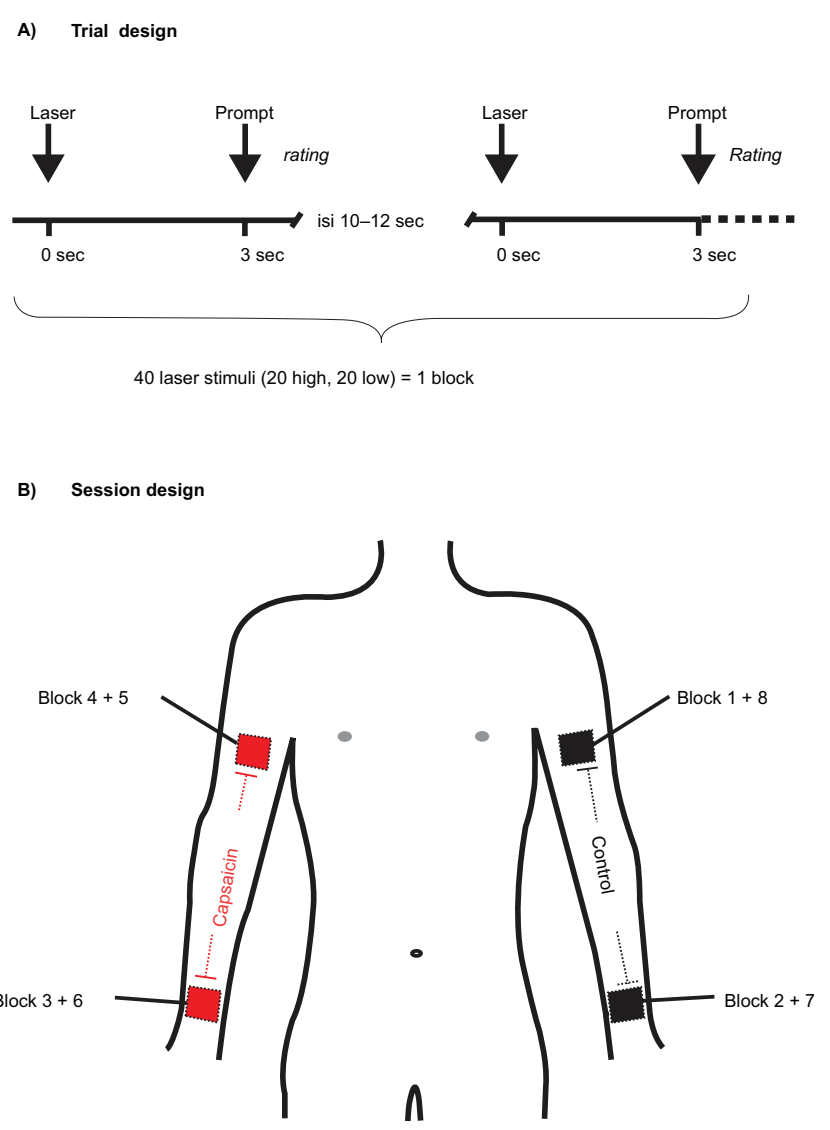

Figure I The test procedure comprised eight blocks consisting of 40 laser stimuli of two intensities. Section $\mathbf{A}$ ) indicates the trial design. The laser was given at time point zero of each trial.Three seconds after the pain stimulus a tone prompted the subjects to verbally rate the perceived pain. Section $\mathbf{B}$ ) shows the experimental procedure of all eight blocks. One arm (left or right, counterbalanced across subjects) was treated proximally as well as distally with topical capsaicin ( $0.1 \%$ dissolved in $70 \%$ ethyl alcohol). The session started with stimulation of the proximal site of the control arm (Block I) and proceeded with Block 2 at the distal control site (distance between proximal and distal sites $=30 \mathrm{~cm}$ ). Laser stimuli of Block 3 were applied to the capsaicin treated distal forearm, followed by Block 4 at the capsaicin treated proximal upper arm. I repetition, the sites were stimulated in reverse block order, thus started at the proximal capsaicin-treated site and ended at the proximal control site. This procedure minimized confounding effects of site with habituation.
Singen, Germany) with two Neuroscan ${ }^{\circledR} 32$ channel synamps at a sampling frequency of $1000 \mathrm{~Hz}$, a $500 \mathrm{~dB}$ gain and a band pass filter between 0.15 and $100 \mathrm{~Hz}$. The electrode impedance was kept below $5 \mathrm{k} \Omega$. The EEG was recorded with reference to linked earlobes. The continuous data sets were segmented in epochs ranging from -1000 to $3000 \mathrm{~ms}$. EEG data were pre-processed offline to remove artifacts using EEGLAB, ${ }^{23} \mathrm{a}$ freely available open source toolbox (http://www.sccn.ucsd. edu/eeglab) running under Matlab (The Mathworks, Natick, MA). Artifact removal was done, first, by visual inspection of all segments for the presence of gross artifacts such as muscular contractions or head movements. Then, data were submitted to extended independent component analysis (ICA). ${ }^{24,25}$ Briefly, ICA returns a set of spatial filters, which, when multiplied with the data, yield component activations being maximally temporally independent from each other. By visual inspection of component maps and component time courses, we identified those independent components reflecting eye blinks and other ocular artifacts ${ }^{26,27}$ and subtracted them from the data. Back-projection of the remaining components revealed EEG data corrected for artifacts, which were then averaged separately for the conditions of interest. We merged trials containing stimuli of both intensities and repetitions delivered to the same location for further time frequency analysis.

\section{Computation of the time frequency representation (TFR)}

For lower frequencies up to $10 \mathrm{~Hz}$, the time frequency transformation was performed by a sliding window Fourier analysis using a Hanning window. For frequencies above $10 \mathrm{~Hz}$, the data were multiplied by $\mathrm{N}>1$ orthogonal tapers and Fourier transformed and the $\mathrm{N}$ spectral estimates were finally averaged. The spectra for each individual taper were magnitude squared after Fourier transformation for power estimation. As data tapers, we used the leading 2TW-1 prolate spheroidal (slepian) sequences, where $\mathrm{T}$ denotes the length of the tapers and $\mathrm{W}$ the half bandwidth. These tapers optimally concentrate the spectral energy of the signal over the desired half bandwidth $\mathrm{W}^{28}$ To obtain phase-locked and non-phaselocked information, total power TFR and evoked power TFR were calculated separately. For total power TFR, spectral power was computed for each single trial before averaging. Thus, all spectral estimates for the total power contained signal components that are non-phase-locked as well as phase-locked to stimulus onset. ${ }^{15,20}$ For the evoked power TFR, the averaged evoked potential was transformed into the frequency domain, thus containing phase-locked information 
only. Spectral amplitudes were computed as the square root of the spectral power estimate. In order to characterize the temporal profile of spectral responses, we performed for all time frequency transformations a sliding window analysis. A window of $200 \mathrm{~ms}$ length for higher frequencies above $10 \mathrm{~Hz}$ and a $400 \mathrm{~ms}$ window for lower frequencies was shifted over the data with a window step size of $5 \mathrm{~ms}$. For frequencies above $10 \mathrm{~Hz}$, spectral smoothing of $10 \mathrm{~Hz}$ was achieved by using 3 slepian tapers. The baseline spectrum was estimated as the average spectrum of the time frequency transform across the interval from $-1000 \mathrm{~ms}$ to $-200 \mathrm{~ms}$ before the stimulus onset. For both total and evoked power, normalization was done by calculating the percentage change of signal amplitude relative to pre-stimulus baseline of the total power. Further analysis was performed using data from a central region of interest (ROI) because the signals and different components had the best signal quality in this central ROI. Lateralized ROIs were also analyzed, but did not reveal new or different results. The central ROI consisted of the vertex position $(\mathrm{Cz})$ and four surrounding electrodes (C1, C2, CPz, FCz).

\section{Statistical analysis}

For statistical analysis, SPSS 10.0 was used (SPSS Inc., Chicago, IL). The critical $p$-value for all analyses was 0.05 . All variables were first tested with a one-sample Kolmogorov-Smirnov test for normal distribution. As no deviations from the normal distribution were detected, we used parametrical statistics. Pain ratings were analyzed using repeated measures 2-way analyses of variance (ANOVA) testing for the effects of stimulus intensity (450 versus $600 \mathrm{~mJ}$ ) and condition (sensitized versus normal). TFRs were analyzed for the effect of skin condition only using a paired t-test. Neither the effects of intensity nor its interaction with condition were tested because the computation of the TFR required a sufficient number of trials, so that we included both the 450-mJ- and 600-mJ-trials in one condition. The identification of the time frequency windows subjected to further analysis was based on the TFR plots. In the respective windows the maximal (for power increase) and the minimal values (for power decrease) were determined for each subject and statistically analyzed. The data processing flow is displayed in Figure 2.

\section{Results}

\section{Psychophysics}

Topical application of capsaicin had a clear sensitization effect as indicated by primary hyperalgesia, mechano-allodynia, redness and hyperthermia on the treated skin area in all
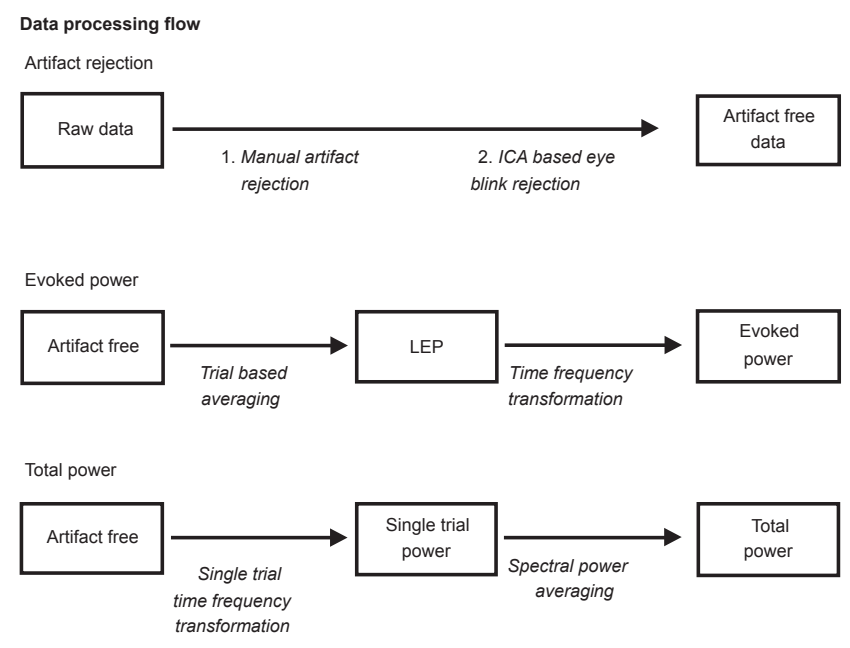

Figure 2 Overview of the data processing flow starting with two steps of artifact elimination procedures applied to the raw data followed by the indicated steps of time frequency transformations to achieve evoked and totalTFR plots. The computation of the evoked TFR also included the averaging of the conventional LEP.

Abbreviations: LEP, laser-evoked potentials; TFR, time frequency representation.

subjects. Furthermore, an ongoing pain sensation occurred within 10 minutes after capsaicin application that lasted for approximately 20 minutes. With the beginning of the experimental blocks, ongoing pain had fully disappeared. Some subjects reported a feeling of warmth that lasted for some time longer and disappeared during the first blocks performed at the control arm. The intensity of the laser stimulus had a significant effect on pain ratings $(\mathrm{F}(1,15)=88.5$; $\mathrm{p}<0.001)$ due to lower ratings after low intensity $(41 \pm 12)$ compared with high intensity stimulation (56 \pm 12$)$. Neither a main effect nor an interaction with intensity was found for condition (capsaicin treated vs. untreated skin). Furthermore, condition had no significant effects regarding the unpleasantness ratings reported after each block.

\section{Phase-locked oscillatory responses}

The grand average TFR of the EEG activity following laser stimuli revealed a dramatic difference between evoked and total TFRs (Figure 3). An early increase of the evoked power in the delta-theta band, subsequently referred to as evoked response I (ER I), appeared between stimulus onset and $500 \mathrm{~ms}$ post-stimulus and spanned frequencies from 3 to $7 \mathrm{~Hz}$. The ER I did not exhibit any significant latency shift between proximal and distal stimulation. Skin sensitization caused a decrease of ER I $(\mathrm{t}(15)=-4.6 ; \mathrm{p}<0.001 ; 276 \% \pm 148 \%$ vs $168 \% \pm 93 \%)$. The corresponding plot in the time domain of the grand average evoked potential waveform illustrates the contribution of ER I to the LEP N2-P2 component which was also diminished by capsaicin treatment (Figure 3). A very 
A LEP Cz

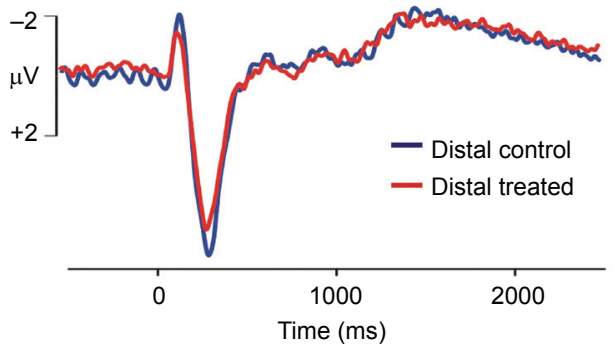

B TFR evoked

TFR total
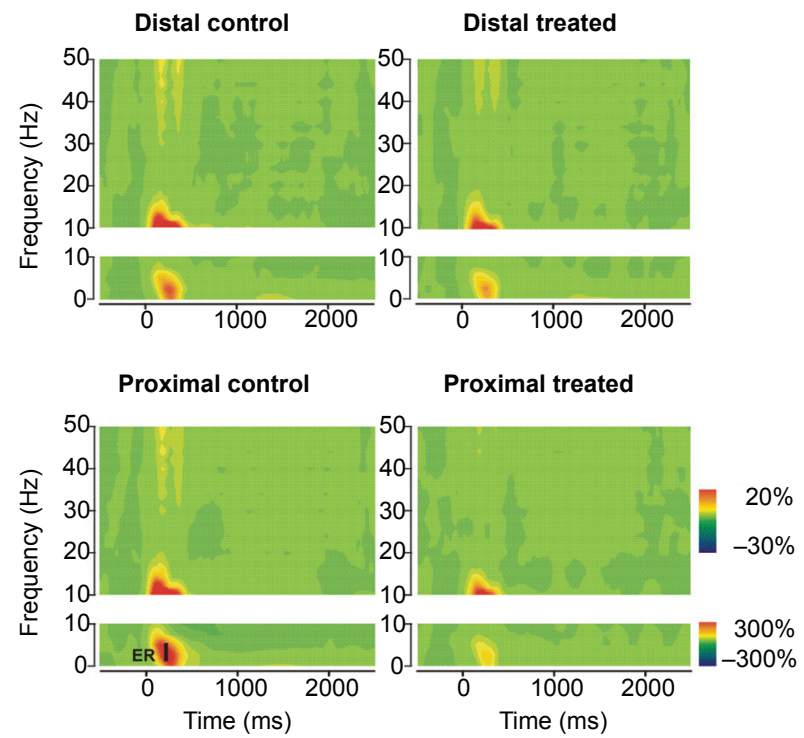
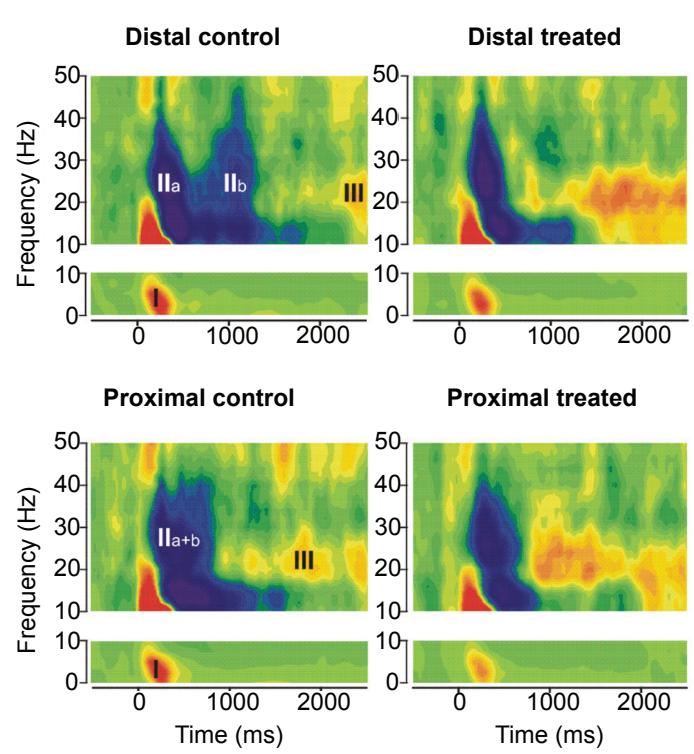

Proximal treated

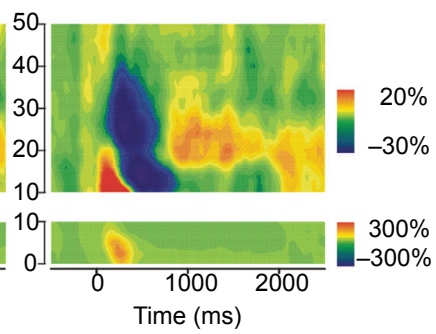

Figure 3 Evoked and total TFR plots illustrating the effects of topical capsaicin treatment at distal and proximal stimulus sites. For comparison, the LEP at central electrode $\mathrm{Cz}$ obtained from stimulation at distal treated and control sites is also shown top left. Time frequency data represent responses from central ROls averaged across all subjects $(\mathrm{N}=16)$. They are plotted as percentage change of signal amplitude relative to pre-stimulus baseline $(-1000$ to $-200 \mathrm{~ms})$. A) Phase-locked evoked response increase (ER I: 3-7 Hz, 0-500 ms) is diminished by skin sensitization as is the N2-P2 component of the LEP. B) Total TFR also reveals a stimulus-induced increase from 3 to $7 \mathrm{~Hz}$ (TR I), which was followed by an alpha-to-gamma decrease (TR II) around I000 ms (distal). This decrease has two minima (TR Ila, TR IIb), the latter delayed as predicted by $\mathrm{C}$-fiber conduction velocity between proximal and distal stimulus sites and suppressed by topical capsaicin treatment. A later upper beta increase (TR III: I5-30 $\mathrm{Hz}$, $1400-2100 \mathrm{~ms}$, distal) also shows a shift in latency between proximal and distal stimulation that is compatible with C-fiber conduction velocity. It exhibits an enhancement following capsaicin treatment.

Abbreviations: LEP, laser-evoked potentials; ROI, region of interest;TFR, time frequency representation.

similar oscillatory response appeared also in the total TFR, which we label TR I (total response I, $\mathrm{t}(15)=-4.2 ; \mathrm{p}=0.001$; $436 \% \pm 200 \%$ vs $294 \% \pm 120 \%)$. The similarity of the frequency characteristics and the peak latency suggests that TR I largely corresponds to ER I, ie, mostly to phase-locked oscillatory activity.

\section{Non-phase-locked oscillatory responses}

Several oscillatory response patterns occurred in the total TFR, but not in the evoked TFR, suggesting the presence of oscillatory activity, which is not phase locked to the stimulus. In contrast to the evoked TFR, the total TFR showed a prominent decrease of power between 10 and $40 \mathrm{~Hz}$, referred to as total response II (TR II). Notably, this power decrease in the alpha-to-gamma frequency range consisted of two separate minima for distal stimulation (TR IIa: 100-500 ms, TR IIb: 900-1300 ms), which merged to one broader response after proximal stimulation (100-900 ms). This phenomenon suggests that TR IIa may be elicited by rapidly conducting A-fibers, whereas slowly conducting $\mathrm{C}$-fibers may give rise to response TR IIb. The latency shift is consistent with C-fiber conduction velocity $(0.3 \mathrm{~m} / 400 \mathrm{~ms}=0.75 \mathrm{~m} / \mathrm{s})$. Interestingly, skin sensitization did not change TR IIa $(\mathrm{t}(15)=1, \mathrm{p}=0.357)$, but significantly attenuated the power decrease of the TR IIb (t $(15)=2.5 ; \mathrm{p}=0.024 ;-35 \% \pm 10 \%$ vs $-29 \% \pm 14 \%$ ). Moreover, the total TFR displayed a power increase at a frequency from 15 to $30 \mathrm{~Hz}$, referred to as total response III (TR III). This latter activity also occurred approximately $400 \mathrm{~ms}$ 
earlier for laser stimulation of the proximal (1000-1700 ms) compared to the distal site (1400-2100 ms). Capsaicin treatment significantly augmented this putative C-fiber-related TR III (t(15) $=2.5 ; \mathrm{p}=0.027 ; 27 \% \pm 17 \%$ vs $36 \% \pm 16 \%)$.

To sum up, three significant pain-related responses were identified by the analysis of evoked and total TFR following painful laser stimuli (Figure 4). First, a power increase in the delta-theta band, mediated by A-fibers, appeared in both the evoked and the total TFR (ER I $\approx$ TR I) and was reduced by topical capsaicin treatment. Second, a broad power decrease ranging from the alpha to the gamma band appeared only in the total TFR (TR II), suggesting an exclusive reduction of non-phase-locked oscillatory activity within this bandwidth. Comparison of proximal and distal stimulus sites suggests a contribution of both A- (TR IIa) and C-nociceptor (TR IIb) activation whereas only the latter was reduced by capsaicin treatment. Third, the total TFR, but not the evoked TFR revealed a power enhancement of an upper beta band oscillation (TR III) that is most likely mediated by $\mathrm{C}$-nociceptors and that was augmented by capsaicin treatment.

\section{Discussion}

The present study explores the presence of separable correlates of A- and C-nociceptor activity in the human EEG of healthy subjects by analyzing phase-locked and non-phase-locked oscillations following painful laser stimuli applied to normal and capsaicin-treated skin at distal and proximal sites. Our results confirm previous observations that C-fiber components are absent in the classical phase-locked LEP when the concomitant A-fiber activation is not blocked or avoided by

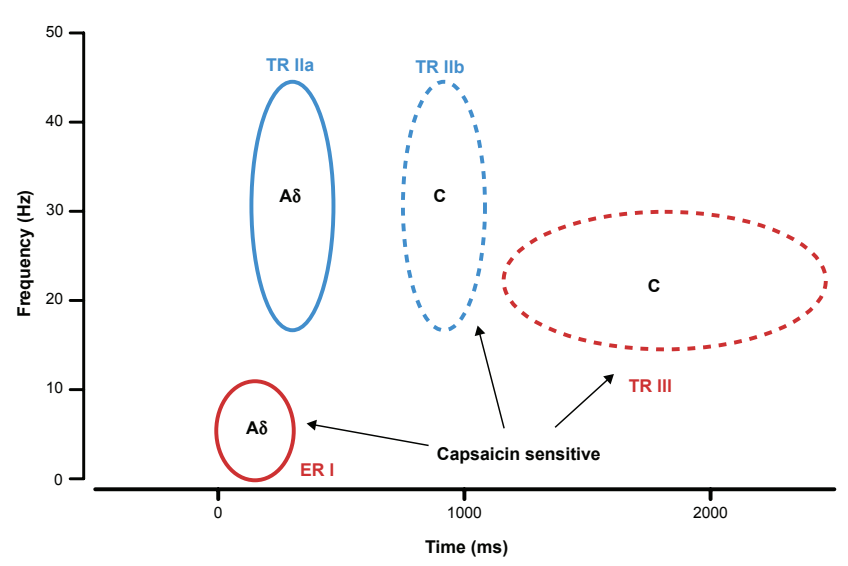

Figure 4 Summary diagram illustrating putative A $\delta$ (solid circles) and C-nociceptor (broken circles) contributions to enhanced (red) and reduced (blue) oscillatory electroencephalography responses in the indicated frequency ranges and their capsaicin sensitivity. special stimulation techniques..$^{9,10,14,16,29}$ Mouraux and colleagues ${ }^{16}$ first pointed to the potential benefit of the analysis of non-phase-locked EEG activity to reveal correlates of C-fiber activation following laser stimuli that they observed in the form of desynchronization of alpha band activity. We extended this approach by application of a multi-taper method for time frequency estimations that is ideally suited for the analysis of higher-frequency oscillations. ${ }^{28}$ It revealed a marked increase of oscillations in the late beta frequency band that appeared exclusively in the total TFR suggesting a purely non-phaselocked activity to the laser stimuli. Based on an adequate latency shift between proximal and distal stimulation sites this oscillation is most likely due to C-fiber activation. However, we did not obtain reaction times or different ratings for the perception of first and second pain to prove if this oscillation that we labeled TR III was indeed C-fiber-related.

Polymodal C-nociceptors express the TRPV1 that gates depolarizing inward currents and renders the fiber more sensitive to heat in response to capsaicin. In contrast, polymodal A-nociceptors, often referred to as type II AMH nociceptors, ${ }^{6}$ exhibit desensitization leading to reduced first pain sensation after topical capsaicin application of much higher concentration (10\%) than used in this study $(0.1 \%) .{ }^{6}$ A reduction of late LEP components as correlates of A-nociceptor activation by topical capsaicin application has also been reported previously. ${ }^{30,31}$ Opposing sensitization and desensitization effects of respective $\mathrm{C}$ - and A-nociceptors may have balanced the psychophysical responses to laser stimuli in this study such that no significant change of subjective painfulness occurred. Yet, desensitization of polymodal A-nociceptors may have contributed to the reduction of the evoked delta power (ER I of evoked TFR) and N2-P2 amplitude of the LEP whereas sensitization of polymodal $\mathrm{C}$-nociceptors may have contributed to the enhancement of the TR III in the total TFR. Notably, the C-fiber-related N2-P2 LEP components following laser stimuli at intensities eliciting just a sensation of warmth were diminished by topical capsaicin, ${ }^{32}$ consistent with the existence of heterogeneous classes of C-fibers in the human skin.

Recent experiments also suggest the importance of a mutual inhibitory interaction at spinal or supraspinal sites of A- and C-nociceptor activation affecting the brain activity induced by either fiber system. ${ }^{33,34}$ Enhanced C-nociceptor background activity following capsaicin sensitization may therefore have contributed to diminished A-nociceptor ER I/TR I activity. In contrast, the dominant feature of first pain elicited by laser stimuli and the fact that it always precedes second pain may be important for the blocking of 
C-fiber correlates in the evoked EEG by A-nociceptor input on normal skin. ${ }^{29}$ Our results therefore shed new light on the phenomenon that A-fiber activity typically "masks" C-fiber correlates in the LEP. Apparently, A-nociceptor input to the brain interferes with the degree of phase-locking of cortical oscillations induced by subsequent $\mathrm{C}$-nociceptor input.

Several authors have argued that given the difference in transduction mechanisms and neurochemical sensitivity, $\mathrm{C}$ - and A-nociceptors likely play different roles in thermosensation and pain. ${ }^{3,35}$ Capsaicin-sensitivity of nociceptors appears to be important for their involvement in inflammatory pain ${ }^{3}$ but not for normal noxious heat sensitivity. ${ }^{4}$ The observation by PET imaging that normal heat pain recruits a largely different supraspinal pain network than equally intense capsaicin-induced heat allodynia/hyperalgesia suggests that a unique class of nociceptors, most likely C-fibers, and specific projection pathways convey information about the status of pathological tissue rather than intact tissue exposed to an acute exteroceptive pain stimulus. $^{7}$ Phase-locking of EEG oscillations to the stimulus onset appears to be a feature of rapid sensory input informing the brain about sudden changes in the environment in order to induce orientation to salient and potentially threatening events. This is quite obviously the dominant function of A-nociceptors, which aside from their supraspinal recruitment of rapid defense behaviors also mediate spinal withdrawal reflexes. $\mathrm{C}$-nociceptors instead provide information about the duration of a sensory event and about the existence of inflammationinduced hypersensitivity in injured tissue. This functional distinction matches the conclusions of Tran and colleagues ${ }^{34}$ that suppressive cortical interaction between A- and C-nociceptor pathways may reflect the activation of contrasting and perhaps mutually exclusive behaviors: an A-nociceptor-mediated channel for rapid escape from threat and a slow $\mathrm{C}$-mediated channel that promotes avoidance and the protection of injured tissue. The existence of robust beta frequency EEG oscillations mediated by $\mathrm{C}$-nociceptors following infrared laser stimuli and their exaggeration by neurogenic inflammation following skin treatment with topical capsaicin suggests a promising approach to probe cortical activity relevant for clinical pain states.

Another interesting observation in this study was the existence of power decreases in a broad frequency spectrum ranging from alpha to beta and gamma bandwidths after painful stimulation. These decreases exhibited two peaks compatible with respective A- and C-nociceptor activations. Mouraux and colleagues ${ }^{16}$ also found a non-phase-locked desynchronization of alpha band activity to follow both A- and $\mathrm{C}$-nociceptor recruitment by laser stimuli. It is possible that this phenomenon reflects the interruption of ongoing oscillations representing "default" maintain pre-stimulus alertness or represent a resting state at the absence of specific sensory input or cognitive activities. Notably, skin sensitization by topical capsaicin strongly attenuated the second peak of the broadband power decrease presumably reflecting a $\mathrm{C}$-nociceptor response, but not the first power decrease associated with A-nociceptor activity. A-nociceptor activation following painful laser ${ }^{18}$ or electrical stimulation ${ }^{19}$ also induces non-phase-locked gamma activity, which can be linked to the internal evaluation of pain. These gamma oscillations can usually be found in high frequencies above $60 \mathrm{~Hz}$ in data recorded using magnetoencephalography (MEG). Due to our filter settings, early gamma oscillations could not reliably be quantified in the present study.

To conclude, the computation of multi-taper based time frequency representations for systematic comparison of phase-locked and non-phase-locked oscillations in the human EEG following repetitive infrared laser stimuli allows the identification of distinct cortical correlates of peripheral A- and C-nociceptor activations without application of special stimulation techniques. Further experiments may test their differential modulation by analgesic pharmacological or psychological interventions or their differential alteration in pathological pain conditions.

\section{Acknowledgments}

Claudia Domnick and Michael Hauck were supported by UKE Junior Research Grant FFM F-161-1 and by the German Research Foundation (SE 1859/1-1). Kenneth L Casey is supported by NIH grant R01 AR46045, the Department of Veteran's Affairs, and a donation to the Veteran's Educational and Research Association of Michigan (Pfizer Co.). Andreas K Engel acknowledges support by the European Commission (grants IST-027268 "Perception on Purpose" and NEST-043457 "Mindbridge") and the Volkswagen Foundation (project "Representation"). Jürgen Lorenz was supported by a grant from the Max-Kade-Foundation.

\section{References}

1. Perl ER. Cutaneous polymodal receptors: characteristics and plasticity. Prog Brain Res. 1996;113:21-37.

2. Meyer RA, Ringkamp M, Campbell JN, Raja SN. Peripheral mechanisms of cutaneous nociception. In: McMahon SB, Koltzenburg M, editors. Textbook of Pain. 5th ed. Philadelphia: Churchill Livingston; 2006. p. 3-34.

3. Caterina MJ, Leffler A, Malmberg AB, et al. Impaired nociception and pain sensation in mice lacking the capsaicin receptor. Science. 2000;288(5464):306-313.

4. Woodbury CJ, Zwick M, Wang S, et al. Nociceptors lacking TRPV1 and TRPV2 have normal heat responses. J Neurosci. 2004;24(28):6410-6415.

5. Tominaga M, Caterina MJ, Malmberg AB, et al. The cloned capsaicin receptor integrates multiple pain-producing stimuli. Neuron. 1998;21(3):531-543. 
6. Magerl W, Fuchs PN, Meyer RA, Treede RD. Roles of capsaicininsensitive nociceptors in cutaneous pain and secondary hyperalgesia. Brain. 2001;124(Pt 9):1754-1764.

7. Lorenz J, Cross D, Minoshima S, Morrow T, Paulson P, Casey K. A unique representation of heat allodynia in the human brain. Neuron. 2002;35(2):383-393.

8. Mouraux A, Plaghki L. Are laser-evoked brain potentials modulated by attending to first or second pain? Pain. 2007;129(3):321-331.

9. Bromm B, Treede RD. Pain related cerebral potentials: late and ultralate components. Int J Neurosci. 1987;33(1-2):15-23.

10. Bragard D, Chen AC, Plaghki L. Direct isolation of ultra-late (C-fiber) evoked brain potentials by $\mathrm{CO} 2$ laser stimulation of tiny cutaneous surface areas in man. Neurosci Lett. 1996;209(2):81-84.

11. Forss N, Raij TT, Seppä M, Hari R. Common cortical network for first and second pain. Neuroimage. 2005;24(1):132-142.

12. Kakigi R, Tran TD, Qiu Y, et al. Cerebral responses following stimulation of unmyelinated C-fibers in humans: electro- and magnetoencephalographic study. Neurosci Res. 2003;45(3):255-275.

13. Opsommer E, Weiss T, Plaghki L, Miltner WH. Dipole analysis of ultralate (C-fibers) evoked potentials after laser stimulation of tiny cutaneous surface areas in humans. Neurosci Lett. 2001;298(1):41-44.

14. Cruccu G, Pennisi E, Truini A, et al. Unmyelinated trigeminal pathways as assessed by laser stimuli in humans. Brain. 2003;126(Pt 10): 2246-2256.

15. Pfurtscheller G, Lopes da Silva FH. Event-related EEG/MEG synchronization and desynchronization: basic principles. Clin Neurophysiol. 1999;110(11):1842-1857.

16. Mouraux A, Guerit JM, Plaghki L. Non-phase locked electroencephalogram (EEG) responses to $\mathrm{CO} 2$ laser skin stimulations may reflect central interactions between $\mathrm{A} \delta$ - and $\mathrm{C}$-fiber afferent volleys. Clin Neurophysiol. 2003;114(4):710-722.

17. Chen AC, Herrmann CS. Perception of pain coincides with the spatial expansion of electroencephalographic dynamics in human subjects. Neurosci Lett. 2001;297(3):183-186.

18. Gross J, Schnitzler A, Timmermann L, Ploner M. Gamma oscillations in human primary somatosensory cortex reflect pain perception. PLoS Biol. 2007;5(5):e133.

19. Hauck M, Lorenz J, Engel AK. Attention to painful stimulation enhances gamma-band activity and synchronization in human sensorimotor cortex. J Neurosci. 2007;27(35):9270-9277.

20. Tallon-Baudry C, Bertrand O. Oscillatory gamma activity in humans and its role in object representation. Trends Cogn Sci. 1999;3(4):151-162.

21. Gross J, Tass PA, Salenius S, Hari R, Freund HJ, Schnitzler A. Cortico-muscular synchronization during isometric muscle contraction in humans as revealed by magnetoencephalography. J Physiol. 2000; (Pt 3):527:623-631.

22. Bauer M, Oostenveld R, Peeters M, Fries P. Tactile spatial attention enhances gamma-band activity in somatosensory cortex and reduces low-frequency activity in parieto-occipital areas. J Neurosci. 2006; 26(2):490-501.
23. Delorme A, Makeig S. EEGLAB: an open source toolbox for analysis of single-trial EEG dynamics including independent component analysis. J Neurosci Methods. 2004;134(1):9-21.

24. Bell AJ, Sejnowski TJ. An information-maximization approach to blind separation and blind deconvolution. Neural Comput. 1995;7(6): 1129-1159.

25. Green JB, Bialy Y, Sora E. High-resolution EEG in poststroke hemiparesis can identify ipsilateral generators during motor tasks. Stroke. 1999;30(12):2659-2665.

26. Debener S, Makeig S, Delorme A, Engel AK. What is novel in the novelty oddball paradigm? Functional significance of the novelty P3 event-related potential as revealed by independent component analysis. Brain Res Cogn Brain Res. 2005;22(3):309-321.

27. Jung TP, Makeig S, Westerfield M, Townsend J, Courchesne E, Sejnowski TJ. Analysis and visualization of single-trial event-related potentials. Hum Brain Mapp. 2001;14(3):166-185.

28. Mitra PP, Pesaran B. Analysis of dynamic brain imaging data. Biophys $J$. 1999;76(2):691-708.

29. Bromm B, Lorenz J. Neurophysiological evaluation of pain. Electroencephalogr Clin Neurophysiol. 1998;107(4):227-253.

30. Beydoun A, Dyke DB, Morrow TJ, Casey KL. Topical capsaicin selectively attenuates heat pain and A delta fiber-mediated laser-evoked potentials. Pain. 1996;65(2-3):189-196.

31. Valeriani M, Arendt-Nielsen L, Le Pera D, et al. Short-term plastic changes of the human nociceptive system following acute pain induced by capsaicin. Clin Neurophysiol. 2003;114(10):1879-1890.

32. Valeriani M, Tinazzi M, Le Pera D, et al. Inhibitory effect of capsaicin evoked trigeminal pain on warmth sensation and warmth evoked potentials. Exp Brain Res. 2005;160(1):29-37.

33. Truini A, Galeotti F, Cruccu G, Garcia-Larrea L. Inhibition of cortical responses to Adelta inputs by a preceding C-related response: testing the "first come, first served" hypothesis of cortical laser evoked potentials. Pain. 2007;131(3):341-347.

34. Tran TD, Matre D, Casey KL. An inhibitory interaction of human cortical responses to stimuli preferentially exciting Adelta or C-fibers. Neuroscience. 2008;152(3):798-808.

35. Ahluwalia J, Rang H, Nagy I. The putative role of vanilloid receptor-like protein-1 in mediating high threshold noxious heatsensitivity in rat cultured primary sensory neurons. Eur J Neurosci. 2002;16(8):1483-1489.

36. Raichle ME, Gusnard DA. Intrinsic brain activity sets the stage for expression of motivated behaviour. J Comp Neurol. 2005;493(1):167-176.

37. Pfurtscheller G. Event-related synchronization (ERS): an electrophysiological correlate of cortical areas at rest. Electroencephalogr Clin Neurophysiol. 1992;83:62-69. 